\title{
DBI analysis of generalised permutation branes
}

\author{
Stefan Fredenhagen and Cosimo Restuccia \\ Max-Planck-Institut für Gravitationsphysik, Albert-Einstein-Institut, \\ D-14424 Golm, Germany \\ E-mail: stefan.fredenhagen@aei.mpg.de, cosimo.restuccia@aei.mpg.de
}

ABSTRACT: We investigate D-branes on the product $G \times G$ of two group manifolds described as Wess-Zumino-Novikov-Witten models. When the levels of the two groups coincide, it is well known that there exist permutation D-branes which are twisted by the automorphism exchanging the two factors. When the levels are different, the D-brane charge group demands that there should be generalisations of these permutation D-branes, and a geometric construction for them was proposed in hep-th/0509153. We give further evidence for this proposal by showing that the generalised permutation D-branes satisfy the DiracBorn-Infeld equations of motion for arbitrary compact, simply connected and simple Lie groups $G$.

Keywords: Brane Dynamics in Gauge Theories, D-branes, Conformal Field Models in String Theory

ArXiv EPRINT: 0908.1049 


\section{Contents}

1 Introduction 1

1.1 DBI analysis 2

2 Coordinates on the group 3

$\begin{array}{ll}2.1 & \text { An example: } \text { coordinates on } \mathrm{SU}(2)\end{array}$

3 The DBI equations of motion $\quad 8$

3.1 The general strategy 8

$\begin{array}{lr}3.2 \text { Target space objects } & 9\end{array}$

$\begin{array}{lll}3.3 & \text { Brane quantities } & 11\end{array}$

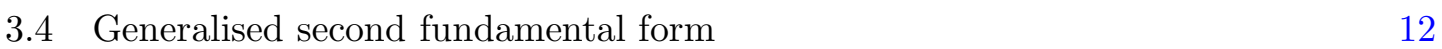

$\begin{array}{ll}\text { 3.5 Checking the equations of motion } & 13\end{array}$

$\begin{array}{ll}\text { 3.5.1 Gauge field equation of motion } & 14\end{array}$

$\begin{array}{ll}\text { 3.5.2 Embedding equations of motion } & 15\end{array}$

4 Further directions $\quad 16$

\section{Introduction}

In background geometries that contain the product of two identical factors, $M \times M$, there exist so-called permutation branes which are $\operatorname{dim} M$-dimensional submanifolds that lie diagonally in the product $M \times M$. On the world-sheet of an open string, they are described by permutation gluing conditions where the chiral left-movers of one factor theory are glued to the corresponding right-moving fields in the second factor and vice versa. When the theory on $M$ is given by a rational conformal field theory (CFT), the boundary states of the permutation branes can be explicitly constructed [1] (see also [2-5]).

It was observed in several instances that these permutation branes play an important role for the D-brane charge group of the background (see e.g. [6-9]). In [10] it was noted that in Gepner models a further construction is needed to explain all the charges, some generalisation of the permutation branes for products of $N=2$ minimal models with different central charges. These have been constructed as matrix factorisations in a Landau-Ginzburg description [10], but no general geometric or boundary conformal field theory construction of these branes is known (see however [11] for the CFT construction in a particular product of minimal models). Earlier there had been a proposal [8] for a generalisation of permutation D-branes in the product of two Wess-Zumino-Novikov-Witten (WZNW) models at different levels, $G_{k_{1}} \times G_{k_{2}}$. Also there it was found that these generalisations are necessary to explain the charge groups predicted by twisted topological K-theory. 
In this note we want to further substantiate the proposal of [8] by verifying that the generalised permutation branes satisfy the Dirac-Born-Infeld (DBI) equations of motion. Such a check has been performed in [8] for the case of $G=S U(2)$, we shall extend it here to arbitrary compact, simply-connected, simple Lie groups $G$.

Let us briefly review the geometry of generalised permutation branes in $G_{k_{1}} \times G_{k_{2}}$ that was suggested in [8]. Write $k_{i}=k_{i}^{\prime} k$ where $k=\operatorname{gcd}\left(k_{1}, k_{2}\right)$ is the greatest common divisor of the levels, so that $k_{1}^{\prime}$ and $k_{2}^{\prime}$ are relatively prime. Then the simplest generalised permutation brane ${ }^{1}$ is described by the embedding

$$
G \ni g \mapsto\left(g^{k_{2}^{\prime}}, g^{-k_{1}^{\prime}}\right) \in G \times G .
$$

This embedding is motivated by the requirement that the $\mathrm{H}$-field on the brane has to be exact: as the H-field is proportional to the level, the induced H-field has vanishing cohomology class on the brane.

We shall check in this paper that the D-brane described by the embedding (1.1) together with the boundary two-form given in (1.5) (the gauge field on the brane) satisfies the DBI equations of motion, and thus - at least in the geometric limit $k \rightarrow \infty$ - defines a consistent D-brane.

The structure of the paper is as follows. In the remainder of the introduction we shall set up the DBI equations of motion in the form that is most useful for us. Section 2 introduces our choice of coordinates on the groups. Finally, the actual computation will be performed in section 3 .

\subsection{DBI analysis}

The dynamics of geometric D-branes are described by the Dirac-Born-Infeld (DBI) theory $[12-14]$. In the next paragraphs we shall review the DBI equations of motion, following the formulation in [15]. Consider a brane that is parameterised by coordinates $x^{a}$ with an embedding in the target space (coordinates $X^{\mu}$ ) given by

$$
x^{a} \mapsto X^{\mu}\left(x^{a}\right)
$$

In our case, the target space index runs over the two group factors $(\mu=1, \ldots, 2 d, d$ is the dimension of each group factor $G$ in the target space), $x^{a}$ will run over the $d$ coordinates of the embedded sub-manifold. We shall distinguish quantities on the brane from target space objects by hats, so $\hat{g}$ and $\hat{B}$ are the induced metric and B-field on the brane, respectively.

Our aim is to verify that the proposed embedding (1.1) minimises the Dirac-Born-Infeld effective action,

$$
S_{\mathrm{DBI}} \propto \int d^{d} x \sqrt{\operatorname{det}(\hat{g}+\hat{B}+\hat{F})},
$$

where $\hat{F}$ is the gauge field strength on the brane. The combined object

$$
\hat{\omega}=\hat{B}+\hat{F}
$$

\footnotetext{
${ }^{1}$ Notice that there are higher dimensional cousins of (1.1), which are not considered in this note.
} 
is the gauge-invariant boundary two-form. For the generalised permutation brane (1.1) it has been proposed in [8] to be (adapted to our normalisation)

$$
\begin{aligned}
\hat{\omega}= & -\frac{k_{1}}{2} \sum_{j=1}^{k_{2}^{\prime}-1}\left(k_{2}^{\prime}-j\right) \operatorname{tr}\left(\operatorname{Ad}_{g^{j}}\left(g^{-1} d g\right) \wedge g^{-1} d g\right) \\
& -\frac{k_{2}}{2} \sum_{j=1}^{k_{1}^{\prime}-1}\left(k_{1}^{\prime}-j\right) \operatorname{tr}\left(\operatorname{Ad}_{g^{-j}}\left(g d g^{-1}\right) \wedge g d g^{-1}\right) .
\end{aligned}
$$

The DBI equations of motion are obtained from a variation of the DBI action (1.3). In a gauge-invariant formulation they read (see [15]),

$$
\begin{aligned}
{\left[(\hat{g}+\hat{\omega})^{-1}\right]^{b a} \Omega_{a b}^{\mu} } & =0 \\
\partial_{a}\left(\sqrt{\operatorname{det}(\hat{g}+\hat{\omega})}\left[(\hat{g}+\hat{\omega})^{-1}\right]_{\text {antisym }}^{a b}\right) & =0 .
\end{aligned}
$$

Here, $\Omega$ is defined to be a generalisation of the second fundamental form,

$$
\Omega_{a b}^{\mu}:=\partial_{a} \partial_{b} X^{\mu}+\Gamma_{\nu \lambda}^{\prime \mu} \partial_{a} X^{\nu} \partial_{b} X^{\lambda}-\hat{\Gamma}_{a b}^{\prime c} \partial_{c} X^{\mu}
$$

with the connections entering this formula being defined in terms of the H-field $H=d B$,

$$
\Gamma^{\prime}:=\Gamma-\frac{1}{2} H
$$

The first equation (1.6) corresponds to a variation of the embedding, the second equation (1.7) comes from the variation of the F-field. For our problem at hand we shall compute the generalised second fundamental form (1.8) explicitly in section 3, with all the geometrical quantities involved. This will then be used to check that the equations (1.6) and (1.7) are indeed satisfied for the generalised permutation branes. ${ }^{2}$ To turn the computation manageable we need to find good coordinates on the brane and on the groups. This will be done in the next section.

\section{Coordinates on the group}

Our analysis starts by choosing a convenient parameterisation of the single group manifold $G$. It was already suggested in [8] that generalised permutation branes are best described with a specific choice of coordinates. These coordinates (described e.g. in [16]) use the triangular decomposition of the Lie algebra; every group element is written as an element of a conjugacy class of an element of the Cartan torus.

A convenient basis for a simple Lie algebra is given by the Cartan-Weyl basis that consists of $r$ (the rank of the algebra) commuting generators $H_{i}$ of the Cartan subalgebra, and

\footnotetext{
${ }^{2}$ In [8] it was already argued that (1.7) holds for arbitrary simple Lie groups. The argument given there, however, was not completely correct and missed out a subtle point. See also footnote 3 on page 15.
} 
the ladder operators $E^{\alpha}$ associated to the roots $\alpha$. They satisfy the commutation relations

$$
\begin{aligned}
& {\left[H_{i}, E^{\alpha}\right]=\alpha_{i} E^{\alpha}, \quad\left[H_{i}, H_{j}\right]=0, \quad i, j=1 \ldots r} \\
& {\left[E^{\alpha}, E^{\beta}\right]= \begin{cases}\sum_{i} \alpha_{i} H_{i} & \text { if } \beta=-\alpha \\
N_{\alpha \beta} E^{\alpha+\beta} & \text { if } \alpha+\beta \in \Delta . \\
0 & \text { otherwise }\end{cases} }
\end{aligned}
$$

By $\Delta$ we denoted the set of all roots $\{\alpha\}, N_{\alpha \beta}$ is some constant. We follow the usual convention that the norm squared of a long root is 2 , and that

$$
\operatorname{tr}\left(H_{i} H_{j}\right)=\delta_{i j}, \quad \operatorname{tr}\left(E^{\alpha} E^{\beta}\right)=\delta^{\alpha,-\beta} .
$$

Now let us choose our parameterisation. Following [16] we write

$$
g(\chi, \theta):=h^{-1}(\theta) t(\chi) h(\theta)
$$

where $t$ is an element of the Cartan torus $T, t(\chi)=\exp \left(i H_{j} \chi^{j}\right)$, with $j$ running from 1 to $r$, and $h \in G$ is only defined up to left translations by $T$ (so it really lives in the quotient space $G / T)$.

One of the beautiful features of this parameterisation is that it allows us to compute quite simply powers of a generic group element,

$$
g^{n}=h^{-1} t^{n} h .
$$

This is particularly useful for the description of generalised permutation branes, because the embedding (1.1) involves powers of group elements.

To complete the parameterisation we need in addition to the coordinates $\chi$ on the Cartan torus to specify good coordinates on the quotient space $G / T$. Following [16] we first introduce the one forms $\theta^{\alpha}$ by decomposing $d h h^{-1}$ in the Cartan-Weyl basis,

$$
d h h^{-1}=\sum_{\alpha>0} i\left[\theta^{\alpha} E^{\alpha}+\theta^{-\alpha} E^{-\alpha}\right]+i \zeta^{i} H_{i}
$$

Note that under a change of a representative $h$ of $G / T, h \rightarrow h^{\prime}=f h$ with $f=\exp \left(i \phi^{j} H_{j}\right) \in$ $T$, the decomposition (2.6) changes to

$$
d h^{\prime} h^{\prime-1}=\sum_{\alpha>0} i\left[e^{i \alpha \cdot \phi} \theta^{\alpha} E^{\alpha}+e^{-i \alpha \cdot \phi} \theta^{-\alpha} E^{-\alpha}\right]+i\left(\zeta^{i}+d \phi^{i}\right) H_{i} .
$$

So we see that the $\theta^{\alpha}$ only change by phases, whereas the torus part $\zeta$ remains invariant only under constant rotations $f$. Up to the problem with the phases, the one-forms $\theta^{\alpha}$ are well-defined objects on $G / T$, they will be used to locally introduce coordinates on $G / T$.

We can express the metric and the H-field on the group by the coordinates $\chi$ and the one-forms $\theta^{\alpha}$. Following again $[8,16]$, on a single group factor $G$ at level $k$ one finds

$$
\begin{aligned}
d s^{2} & =-\frac{k}{2} \operatorname{tr}\left[g^{-1} d g \otimes_{s} g^{-1} d g\right] \\
& =\frac{k}{2}\left[\sum_{\alpha>0} 4 \sin ^{2} \frac{\chi^{i} \alpha_{i}}{2}\left[\theta^{\alpha} \otimes \theta^{-\alpha}+\theta^{-\alpha} \otimes \theta^{\alpha}\right]+\sum_{j=1}^{r} d \chi^{j} \otimes d \chi^{j}\right] .
\end{aligned}
$$


The phase ambiguity of the $\theta^{\alpha}$ obviously drops out, because $\theta^{\alpha}$ and $\theta^{-\alpha}$ change by opposite phases. We can also express the $\mathrm{H}$-field in this parameterisation,

$$
H=d B=k d\left[i \sum_{\alpha>0}\left(\chi^{j} \alpha_{j}-\sin \chi^{j} \alpha_{j}\right) \theta^{\alpha} \wedge \theta^{-\alpha}\right] .
$$

Note that again the phase ambiguity in the $\theta^{\alpha}$ drops out. As $H$ is not exact, the B-field defined by (2.10) is not globally well-defined, it is $\operatorname{singular}$ at those $t=\exp (i H \cdot \chi) \in T$ different from the identity, whose conjugacy class degenerates (and thus has dimension smaller than $G / T)$.

It is instructive to see how the parameterisation in terms of $\chi$ and the $\theta$ 's depend on the choice of a Cartan torus $T$. Suppose we choose a different torus $T^{\prime}$. By a well-known theorem, the two tori are conjugate (see e.g. [17, Chapter IV, Theorem 1.6]), and there is a $g_{0} \in G$ with

$$
T^{\prime}=g_{0} T g_{0}^{-1}
$$

The decomposition of a group element $g$ with respect to $T$ is then related to the one with respect to $T^{\prime}$ in a simple way,

$$
g=h^{-1} t h=\left(g_{0} h\right)^{-1} g_{0} t g_{0}^{-1}\left(g_{0} h\right)=h^{\prime-1} t^{\prime} h^{\prime},
$$

where now $t^{\prime}=g_{0} t g_{0}^{-1}$ and $h^{\prime}=g_{0} h$. To find the coordinates $\chi^{\prime}$ and the one-forms $\theta^{\prime \alpha}$ we have to use the changed basis of generators, $E^{\prime \alpha}=g_{0} E^{\alpha} g_{0}^{-1}$ and $H_{i}^{\prime}=g_{0} H_{i} g_{0}^{-1}$. Then

$$
t^{\prime}=g_{0} t g_{0}^{-1}=g_{0} e^{i \sum H_{j} \chi^{j}} g_{0}^{-1}=e^{i \sum H_{j}^{\prime} \chi^{j}},
$$

so that $\chi^{\prime j}=\chi^{j}$. On the other hand we have

$$
d h^{\prime} h^{\prime-1}=g_{0}\left(d h h^{-1}\right) g_{0}^{-1}=\sum_{\alpha>0} i\left[\theta^{\alpha} E^{\prime \alpha}+\theta^{-\alpha} E^{\prime-\alpha}\right]+i \zeta^{i} H_{i}^{\prime},
$$

so that $\theta^{\prime \alpha}=\theta^{\alpha}$. The parameterisation is therefore independent of the choice of the Cartan torus.

We have seen that the parameterisation (2.4) in terms of $\chi$ and $\theta$ has very nice properties. On the other hand we still face the problem that $\theta^{\alpha}$ are just one-forms and not yet coordinates. If they were exact, we could introduce coordinates by setting $\theta^{\alpha}=d z^{\alpha}$. However, as we shall see shortly, the one-forms $\theta^{\alpha}$ are not even closed. Still it is possible to introduce coordinates $z$ locally around $g=t(\chi)$ that capture all of the nice features and satisfy

$$
\theta^{\alpha}=d z^{\alpha}+\mathcal{O}(z)
$$

Namely we shall parameterise $h$ by

$$
h=e^{i\left(\sum_{\alpha \in \Delta} z^{\alpha} E^{\alpha}\right)}=1+i \sum_{\alpha \in \Delta} z_{\alpha} E^{\alpha}+\mathcal{O}\left(z^{2}\right) .
$$

To get the one-forms $\theta^{\alpha}$ in terms of the variables $z$, we expand $d h h^{-1}$ in $z$, $d h h^{-1}=\sum_{\alpha, \beta \in \Delta^{+}}\left[\left(i E^{\alpha} d z^{\alpha}-\frac{1}{2}\left[E^{\alpha}, E^{\beta}\right] d z^{\alpha} z^{\beta}\right)+\left(i E^{-\alpha} d \bar{z}^{\alpha}-\frac{1}{2}\left[E^{-\alpha}, E^{-\beta}\right] d \bar{z}^{\alpha} \bar{z}^{\beta}\right)\right]+\mathcal{O}\left(z^{2}\right)$. 
By $\Delta^{+} \subset \Delta$ we denoted the set of all positive roots. From this expression we can extract the one-forms $\theta^{\alpha}, \theta^{-\alpha}$ by using (2.6) and (2.3),

$$
\begin{aligned}
i \theta^{\alpha} & =\operatorname{tr}\left(E^{-\alpha} d h h^{-1}\right)=i d z^{\alpha}-\frac{1}{2} d z^{\beta} z^{\gamma} \operatorname{tr}\left(E^{-\alpha}\left[E^{\beta}, E^{\gamma}\right]\right)+\mathcal{O}\left(z^{2}\right), \\
i \theta^{-\alpha} & =\operatorname{tr}\left(E^{\alpha} d h h^{-1}\right)=i d \bar{z}^{\alpha}-\frac{1}{2} d \bar{z}^{\beta} \bar{z}^{\gamma} \operatorname{tr}\left(E^{\alpha}\left[E^{-\beta}, E^{-\gamma}\right]\right)+\mathcal{O}\left(z^{2}\right) .
\end{aligned}
$$

We can further simplify the formulae (2.18) using the Cartan-Weyl structure equations (2.2), and using the fact that $N_{-\alpha,-\beta}=-\bar{N}_{\alpha \beta}$,

$$
\begin{aligned}
\theta^{\alpha} & =d z^{\alpha}+\frac{i}{2} \sum_{\beta, \gamma \in \Delta^{+}} N_{\beta \gamma} d z^{\beta} z^{\gamma} \delta_{\beta+\gamma, \alpha}+\mathcal{O}\left(z^{2}\right) \\
\theta^{-\alpha} & =d \bar{z}^{\alpha}-\frac{i}{2} \sum_{\beta, \gamma \in \Delta^{+}} \bar{N}_{\beta \gamma} d \bar{z}^{\beta} \bar{z}^{\gamma} \delta_{\beta+\gamma, \alpha}+\mathcal{O}\left(z^{2}\right) .
\end{aligned}
$$

We see now explicitly that the $\theta^{\alpha}$ are in general not closed, even if we sit on the Cartan torus $(z=0)$,

$$
\begin{aligned}
\left.d \theta^{\alpha}\right|_{z, \bar{z}=0} & =-\frac{i}{2} \sum_{\beta, \gamma \in \Delta^{+}} N_{\beta \gamma} \delta_{\beta+\gamma, \alpha} d z^{\beta} \wedge d z^{\gamma} \neq 0 & & \text { in general } \\
\left.d \theta^{-\alpha}\right|_{z, \bar{z}=0} & =\frac{i}{2} \sum_{\beta, \gamma \in \Delta^{+}} \bar{N}_{\beta \gamma} \delta_{\beta+\gamma, \alpha} d \bar{z}^{\beta} \wedge d \bar{z}^{\gamma} \neq 0 & & \text { in general. }
\end{aligned}
$$

Expanding the metric (2.9) in $z$ we find

$$
\begin{aligned}
d s^{2}= & \frac{k}{2}\left[\sum_{\alpha \in \Delta^{+}} 4 \sin ^{2} \frac{\chi^{i} \alpha_{i}}{2}\left[d z^{\alpha} \otimes d \bar{z}^{\alpha}+d \bar{z}^{\alpha} \otimes d z^{\alpha}\right]+\sum_{j=1}^{r} d \chi^{j} \otimes d \chi^{j}\right] \\
& +\frac{k}{4}\left[\sum_{\alpha, \beta, \gamma>0} 4 \sin ^{2} \frac{\chi^{i} \alpha_{i}}{2} \delta_{\beta+\gamma, \alpha}\left[i N_{\beta \gamma} z^{\gamma}\left(d z^{\beta} \otimes_{s} d \bar{z}^{\alpha}\right)-i \bar{N}_{\beta \gamma} \bar{z}^{\gamma}\left(d z^{\alpha} \otimes_{s} d \bar{z}^{\beta}\right)\right]\right] \\
& +\mathcal{O}\left(z^{2}\right) .
\end{aligned}
$$

The first line of the previous expression is a real quadratic form; the second line involves a hermitian bilinear, as one can easily check. The H-field on the other hand takes the following form:

$$
\begin{aligned}
H= & k d\left[i \sum_{\alpha>0}\left(\chi^{j} \alpha_{j}-\sin \chi^{j} \alpha_{j}\right) d z^{\alpha} \wedge d \bar{z}^{\alpha}\right] \\
& +\frac{i k}{2} d\left[\sum_{\alpha, \beta, \gamma>0} \delta_{\beta+\gamma, \alpha}\left(\chi^{j} \alpha_{j}-\sin \chi^{j} \alpha_{j}\right)\left[i N_{\beta \gamma} z^{\gamma}\left(d z^{\beta} \wedge d \bar{z}^{\alpha}\right)-i \bar{N}_{\beta \gamma} \bar{z}^{\gamma}\left(d z^{\alpha} \wedge d \bar{z}^{\beta}\right)\right]\right] \\
& +\mathcal{O}\left(z^{2}\right) .
\end{aligned}
$$




\subsection{An example: coordinates on $\mathrm{SU}(2)$}

We shall illustrate our choice of coordinates in the case of $\mathrm{SU}(2)$. Consider a standard parameterisation of $\mathrm{SU}(2)$,

$$
g(\psi, \vartheta, \phi)=\left(\begin{array}{cc}
\cos \psi+i \cos \vartheta \sin \psi & \sin \psi \sin \vartheta e^{i \phi} \\
-\sin \psi \sin \vartheta e^{-i \phi} & \cos \psi-i \cos \vartheta \sin \psi
\end{array}\right)=h(\vartheta, \phi) t(\psi) h(\vartheta, \phi)^{-1},
$$

with

$$
\begin{aligned}
t(\psi) & =\left(\begin{array}{cc}
e^{i \psi} & 0 \\
0 & e^{-i \psi}
\end{array}\right), \\
h(\vartheta, \phi) & =\left(\begin{array}{cc}
\cos \frac{\vartheta}{2} & -i \sin \frac{\vartheta}{2} e^{i \phi} \\
-i \sin \frac{\vartheta}{2} e^{-i \phi} & \cos \frac{\vartheta}{2}
\end{array}\right) .
\end{aligned}
$$

Using the standard form for the generators,

$$
E^{+}=\left(\begin{array}{ll}
0 & 1 \\
0 & 0
\end{array}\right), \quad E^{-}=\left(\begin{array}{ll}
0 & 0 \\
1 & 0
\end{array}\right),
$$

we can extract the one-forms $\theta^{+}$and $\theta^{-}$from (2.6) and find

$$
\theta^{+}=-\frac{1}{2} e^{i \phi} d \vartheta-\frac{i}{2} \sin \vartheta e^{i \phi} d \phi, \quad \theta^{-}=\overline{\theta^{+}} .
$$

Let us now identify the $z, \bar{z}$ variables in this simple case. Following the definition (2.16) we write

$$
h=e^{i\left(z E^{+}+\bar{z} E^{-}\right)}=\left(\begin{array}{cc}
\cos |z| & i \frac{z}{|z|} \sin |z| \\
i \frac{\bar{z}}{|z|} \sin |z| & \cos |z|
\end{array}\right) .
$$

The connection of the coordinates $z, \bar{z}$ to the old parameterisation is given by

$$
z=-\frac{\vartheta}{2} e^{i \phi}
$$

and the one-forms $\theta^{+}, \theta^{-}$(see $\left.(2.30)\right)$ read now

$$
\begin{aligned}
\theta^{+} & =\left(\frac{1}{2}+\frac{1}{4} \frac{\sin 2|z|}{|z|}\right) d z+\left(\frac{1}{2}-\frac{1}{4} \frac{\sin 2|z|}{|z|}\right) \frac{z}{\bar{z}} d \bar{z} \\
& =d z+\frac{1}{3}\left(z^{2} d \bar{z}-|z|^{2} d z\right)+\mathcal{O}\left(|z|^{4}\right),
\end{aligned}
$$

and $\theta^{-}=\overline{\theta^{+}}$. These one-forms are not closed,

$$
d \theta^{+}=\frac{\sin ^{2}|z|}{\bar{z}} d z \wedge d \bar{z},
$$

but $d \theta^{+}$vanishes at $z=0$. In contrast, for a generic Lie group, $d \theta^{\alpha}$ does not have to vanish at $z=0$ (see $(2.22),(2.23)$ ). This is because in $S U(2)$ there are no non-trivial relations among the positive roots, as there is only one. 


\section{The DBI equations of motion}

We now want to check the DBI equations of motion. As the computations are rather involved, we shall first outline the general strategy.

\subsection{The general strategy}

To check the equations of motion for the generalised permutation branes at a given point of the brane parameterised by $g \in G$, we choose a Cartan torus $T$ containing $g$. (This is always possible, see e.g. [17].) Then we can introduce coordinates $\chi^{j}$ and $z^{\alpha}$ locally around $g$ as in section 2. We want to check the equations at $g$, that is at $z^{\alpha}=0$, but as the equations of motion contain also first derivatives of the geometric data (metric, B-field), we have to keep terms up to linear order in $z$. The function describing the embedding (1.1) enters even with second derivatives, but it can be chosen such that it is exactly linear and its second derivatives vanish,

$$
(\chi, z) \mapsto\left(\left(k_{2}^{\prime} \chi, z\right),\left(-k_{1}^{\prime} \chi, z\right)\right) .
$$

This simple form of the embedding will in particular be very practical when computing the connections.

The computations, although rather involved, simplify because of two reasons: firstly, the target space is a direct product of two - up to the level - identical factors, so that the target space data has a natural block structure. Secondly, our choice of the coordinate system allows us to factorise the geometry in a toroidal part (the directions belonging to the Cartan torus), and a non-toroidal part. We can then introduce "matryoshka" matrices, namely blocks of block matrices such that many of the blocks are trivial and the computations can be reduced considerably.

Before we start with the computation, let us introduce some notations and conventions. For the brane we shall use the coordinates

$$
\left(x^{a}\right)=\left(\chi^{i}, z^{m}, \bar{z}^{m}\right)
$$

where the first $r=\operatorname{rank} G$ coordinates $x^{a}$ denote the toroidal parameters $\chi^{i}$ followed by the remaining $d-r$ coordinates corresponding to the $d-r$ roots. Here we introduced a numbering of the positive roots $\alpha^{m}$ by a label $m$, and we shall often write $z^{m}$ instead of $z^{\alpha^{m}}$.

Similarly, we parameterise the target space by coordinates $X^{\mu}$ where for $\mu=1, \ldots, d$ they denote the coordinates $\left(\chi_{(1)}^{i}, z_{(1)}^{m}, \bar{z}_{(1)}^{m}\right)$ of the first group factor, and for $\mu=d+1, \ldots, 2 d$ they are given by the coordinates of the second group factor. Hats ${ }^{\wedge}$ denote quantities on the brane, a tilde $\sim$ denotes a geometric object of a single factor of the product target space.

We divide the computations in several steps. First we determine the expressions for the target space data in our coordinate system (section 3.2). Then all quantities on the brane will be determined in section 3.3, and in section 3.4 the second fundamental form will be computed. In section 3.5 the equations (1.7) and (1.6) will be checked showing that the generalised permutation branes are extremal points of the DBI action. 


\subsection{Target space objects}

The target space metric is just given by the metrics (2.24) of the two group factors,

$$
\begin{aligned}
d s^{2}= & \sum_{j=1,2}\left\{\frac{k_{j}}{2}\left[\sum_{\alpha>0} 4 \sin ^{2} \frac{\chi_{(j)}^{i} \alpha_{i}}{2}\left[d z_{(j)}^{\alpha} \otimes d \bar{z}_{(j)}^{\alpha}+d \bar{z}_{(j)}^{\alpha} \otimes d z_{(j)}^{\alpha}\right]+\sum_{l=1}^{r} d \chi_{(j)}^{l} \otimes d \chi_{(j)}^{l}\right]\right. \\
& \left.+\frac{i k_{j}}{4}\left[\sum_{\alpha, \beta, \gamma>0} 4 \sin ^{2} \frac{\chi_{(j)}^{i} \alpha_{i}}{2} \delta_{\beta+\gamma, \alpha}\left[N_{\beta \gamma} z_{(j)}^{\gamma}\left(d z_{(j)}^{\beta} \otimes_{s} d \bar{z}_{(j)}^{\alpha}\right)-\bar{N}_{\beta \gamma} \bar{z}_{(j)}^{\gamma}\left(d z_{(j)}^{\alpha} \otimes_{s} d \bar{z}_{(j)}^{\beta}\right)\right]\right]\right\} \\
& +\mathcal{O}\left(z^{2}\right) .
\end{aligned}
$$

Writing the metric $G_{\mu \nu}$ as a matrix it takes a block form,

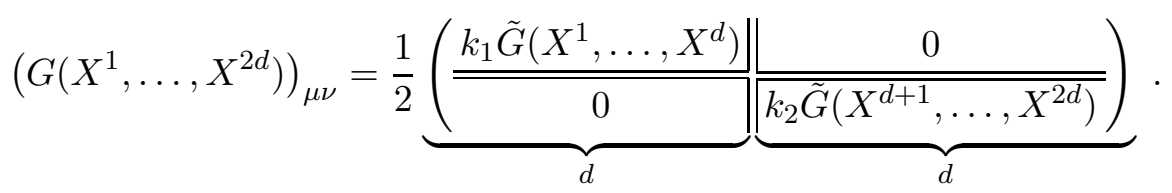

The blocks of $d \times d$-matrices are separated by double lines. The constituent square matrix $\tilde{G}$ has again a block structure with respect to toroidal and non-toroidal directions (indicated by single lines),

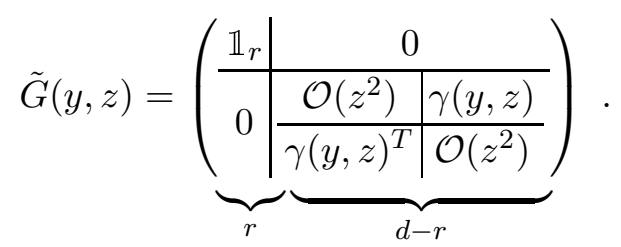

The hermitian square matrix $\gamma$ has size $(d-r) / 2$, and it is given by

$$
\gamma_{m n}(y, z)= \begin{cases}4 \sin ^{2}\left(\frac{y^{k} \alpha_{k}^{n}}{2}\right)+\mathcal{O}\left(z^{2}\right) & \text { for } m=n \\ 2 i \sin ^{2} \frac{\chi^{j} \alpha_{j}^{n}}{2} N_{\alpha^{m}, \alpha^{n}-\alpha^{m}} z^{\alpha^{n}-\alpha^{m}}+\mathcal{O}\left(z^{2}\right) & \text { for } \alpha^{n}-\alpha^{m} \in \Delta^{+} \\ -2 i \sin ^{2} \frac{\chi^{j} \alpha_{j}^{m}}{2} \bar{N}_{\alpha^{n}, \alpha^{m}-\alpha^{n}} \bar{z}^{\alpha^{m}-\alpha^{n}}+\mathcal{O}\left(z^{2}\right) & \text { for } \alpha^{m}-\alpha^{n} \in \Delta^{+} \\ \mathcal{O}\left(z^{2}\right) & \text { otherwise } .\end{cases}
$$

Notice that the diagonal does not have any linear contribution in $z$. Furthermore, the off-diagonal terms are all at least linear in $z$, but $\gamma_{m n}$ does neither depend on $z^{\alpha^{m}}$ nor on $\bar{z}^{\alpha^{m}}$ linearly,

$$
\left.\partial_{a} \gamma_{m n}\right|_{z=0}=0 \quad \text { for } a \cong z^{m}, \bar{z}^{m} .
$$

The background H-field is also just the sum of the H-fields (2.25) of the two factors,

$$
\begin{aligned}
H= & \sum_{j=1,2} \frac{i k_{j}}{2} d\left[\sum_{\alpha>0} 2\left(\chi_{(j)}^{k} \alpha_{k}-\sin \chi_{(j)}^{k} \alpha_{k}\right) d z_{(j)}^{\alpha} \wedge d \bar{z}_{(j)}^{\alpha}\right. \\
& \left.+\sum_{\alpha, \beta, \gamma>0} \delta_{\beta+\gamma, \alpha}\left(\chi_{(j)}^{k} \alpha_{k}-\sin \chi_{(j)}^{k} \alpha_{k}\right)\left[i N_{\beta \gamma} z_{(j)}^{\gamma}\left(d z_{(j)}^{\beta} \wedge d \bar{z}_{(j)}^{\alpha}\right)-i \bar{N}_{\beta \gamma} \bar{z}_{(j)}^{\gamma}\left(d z_{(j)}^{\alpha} \wedge d \bar{z}_{(j)}^{\beta}\right)\right]\right] \\
& +\mathcal{O}(z) .
\end{aligned}
$$


Writing $H$ as $2 d \times 2 d$ matrices $H_{\mu}=\left(H_{\mu \nu \lambda}\right)_{\nu \lambda}$ we have again a block structure,

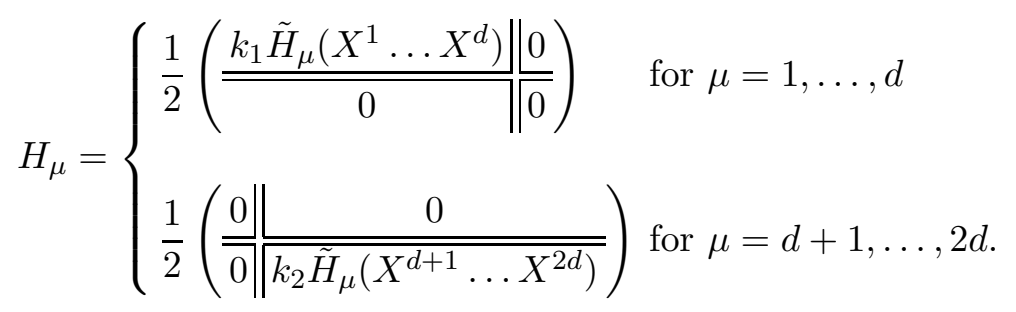

The form of the $d \times d$-matrices $\tilde{H}_{\mu}$ is different for toroidal and non-toroidal directions $\mu$. For toroidal directions we have

$$
\left(\tilde{H}_{\chi^{i}}\right)_{a b}(y, z)=\left(\begin{array}{c|c|c}
0 & \multicolumn{2}{|c}{0} \\
\hline 0 & \mathcal{O}(z) & \beta,{ }_{i}(y, z) \\
\hline-\beta,_{i}^{T}(y, z) & \mathcal{O}(z)
\end{array}\right), \quad \begin{aligned}
& i=1 \ldots r \\
& a, b=1 \ldots d
\end{aligned}
$$

Here we have introduced the anti-hermitian matrix $\beta$ of size $(d-r) / 2$,

$$
\beta_{m n}(y, z)= \begin{cases}2 i\left(y^{k} \alpha_{k}^{n}-\sin y^{k} \alpha_{k}^{n}\right)+\mathcal{O}\left(z^{2}\right) & \text { for } m=n \\ -\left(y^{k} \alpha_{k}^{n}-\sin y^{k} \alpha_{k}^{n}\right) N_{\alpha^{m}, \alpha^{n}-\alpha^{m}} z^{\alpha^{n}-\alpha^{m}}+\mathcal{O}\left(z^{2}\right) & \text { for } \alpha^{n}-\alpha^{m} \in \Delta^{+} \\ \left(y^{k} \alpha_{k}^{m}-\sin y^{k} \alpha_{k}^{m}\right) \bar{N}_{\alpha^{n}, \alpha^{m}-\alpha^{n}} \bar{z}^{\alpha^{m}-\alpha^{n}}+\mathcal{O}\left(z^{2}\right) & \text { for } \alpha^{m}-\alpha^{n} \in \Delta^{+} \\ \mathcal{O}\left(z^{2}\right) & \text { otherwise }\end{cases}
$$

and its derivatives $\beta,_{i}(y, z):=\partial_{i} \beta(y, z)$. The matrix $\beta$, similarly to $\gamma$, has linear terms in $z$ only in off-diagonal terms, and it enjoys a analogous property to (3.7),

$$
\left.\partial_{a} \beta_{m n}\right|_{z=0}=0 \quad \text { for } a \cong z^{m}, \bar{z}^{m} .
$$

For the non-toroidal directions it will be sufficient for the computation to observe that $\tilde{H}$ has the form

$$
\left(\tilde{H}_{z^{m}}\right)_{a b}(y, z)=\left(\begin{array}{c|c|c}
0 & * \\
\hline * & * & \square \\
\hline \square & *
\end{array}\right)+\mathcal{O}(z) \quad\left(\text { similarly for } \tilde{H}_{\bar{z}^{m}}\right)
$$

where $*$ denotes an arbitrary contribution, whereas $\nabla$ denotes a matrix that has vanishing diagonal, $\nabla_{i j}=0$ for $i=j$.

The only target space data that is still missing for the computation is the connection,

$$
\Gamma_{\mu \lambda \nu}:=\frac{1}{2}\left(\partial_{\lambda} G_{\mu \nu}+\partial_{\nu} G_{\mu \lambda}-\partial_{\mu} G_{\nu \lambda}\right) .
$$

Again we write it in matrix form where it assumes a block structure,

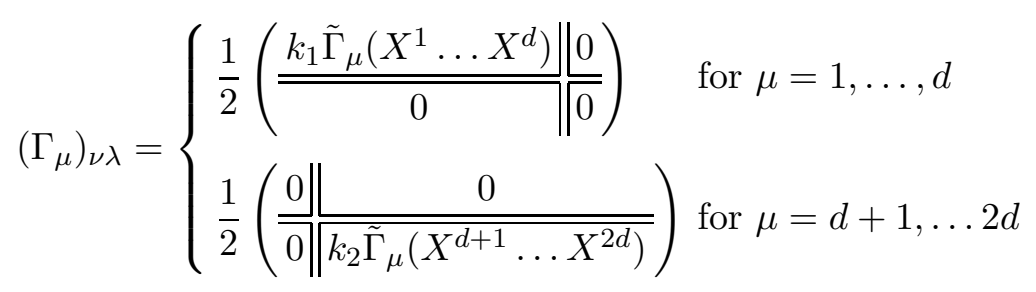


with

$$
\begin{aligned}
& \left(\tilde{\Gamma}_{\chi^{i}}\right)_{a b}(y, z)=-\frac{1}{2}\left(\begin{array}{c|c|c}
0 & \multicolumn{2}{|c}{0} \\
\hline 0 & 0 & \gamma, i(y, z) \\
\hline \gamma, i(y, z) & 0
\end{array}\right)+\mathcal{O}(z), \quad \begin{array}{l}
i=1 \ldots r \\
a, b=1 \ldots d
\end{array} \\
& \left(\tilde{\Gamma}_{z^{m}}\right)_{a b}(y, z)=\left(\begin{array}{c|c|c}
0 & * \\
\hline * & \frac{0}{\square} & \square
\end{array}\right)+\mathcal{O}(z) \quad\left(\text { similarly for } \tilde{\Gamma}_{\bar{z}^{m}}\right),
\end{aligned}
$$

where as before $*$ is not further specified, and $\square$ is a matrix with vanishing diagonal terms.

\subsection{Brane quantities}

The quantities on the world-volume of the brane that are important in our analysis are first of all the induced metric $\hat{g}$ and the boundary two-form $\hat{\omega}$, which we have to determine up to linear order in $z$, and then their derivatives, namely the connection $\hat{\Gamma}$ and the induced $\mathrm{H}$-field $\hat{H}$, for which we only need the expressions at $z=0$.

We start with the induced metric, determined by

$$
\hat{g}_{a b}:=G_{\mu \nu} \partial_{a} X^{\mu} \partial_{b} X^{\nu} .
$$

The contributions from the two factors add up, and we find

$$
(\hat{g})_{a b}(\chi, z)=\left(\begin{array}{c|c}
\frac{\frac{k_{1}^{\prime} k_{2}^{\prime}\left(k_{1}+k_{2}\right)}{2} \mathbb{1}_{r}}{2} & 0 \\
\hline 0 & \frac{1}{2}\left(\begin{array}{c|c}
\mathcal{O}\left(z^{2}\right) & \hat{\gamma}(\chi, z) \\
\hline \hat{\gamma}^{T}(\chi, z) & \mathcal{O}\left(z^{2}\right)
\end{array}\right)
\end{array}\right)
$$

where the matrix $\hat{\gamma}$ of size $(d-r) / 2$ is given in terms of $\gamma$ (defined in (3.6)),

$$
\hat{\gamma}(\chi, z)=k_{1} \gamma\left(k_{2}^{\prime} \chi, z\right)+k_{2} \gamma\left(-k_{1}^{\prime} \chi, z\right) .
$$

The connection $\hat{\Gamma}$ corresponding to the induced metric $\hat{g}$,

$$
\hat{\Gamma}_{c a b}:=\frac{1}{2}\left(\partial_{b} \hat{g}_{c a}+\partial_{a} \hat{g}_{c b}-\partial_{c} \hat{g}_{a b}\right),
$$

is then obtained as

$$
\begin{aligned}
& \left(\hat{\Gamma}_{\chi^{i}}\right)_{a b}=-\frac{k k_{1}^{\prime} k_{2}^{\prime}}{4}\left(\begin{array}{c|c|c}
0 & \multicolumn{2}{|c}{0} \\
\hline 0 & 0 & \gamma, i\left(k_{2}^{\prime} \chi\right)-\gamma,{ }_{i}\left(-k_{1}^{\prime} \chi\right) \\
\hline \gamma, i\left(k_{2}^{\prime} \chi\right)-\gamma, i\left(-k_{1}^{\prime} \chi\right) & 0
\end{array}\right)+\mathcal{O}(z) \\
& \left(\hat{\Gamma}_{z^{m}}\right)_{a b}=\left(\begin{array}{c|c|c}
0 & * \\
\hline * & 0 & \square \\
\hline \square & 0
\end{array}\right)+\mathcal{O}(z) \quad\left(\text { similarly for } \hat{\Gamma}_{\bar{z}^{m}}\right) .
\end{aligned}
$$

In addition to the metric data, we also have to specify the gauge field living on the brane, which is specified by the boundary two-form $\hat{\omega}$ given in (1.5). It is not difficult to show that in terms of $\chi$ and $\theta$ it can be expressed as

$$
\hat{\omega}=i \sum_{\alpha>0}\left(k_{2} \sin k_{1}^{\prime} \chi^{j} \alpha_{j}-k_{1} \sin k_{2}^{\prime} \chi^{j} \alpha_{j}\right) \theta^{\alpha} \wedge \theta^{-\alpha} .
$$


Expressed in the local coordinates $(\chi, z)$ it has the form

$$
(\hat{\omega})_{a b}(\chi, z)=\left(\begin{array}{c|c|c}
0 & 0 \\
\hline 0 & \frac{1}{2}\left(\begin{array}{c|c}
\mathcal{O}\left(z^{2}\right) & \hat{\beta}(\chi, z) \\
\hline-\hat{\beta}^{T}(\chi, z) & \mathcal{O}\left(z^{2}\right)
\end{array}\right)
\end{array}\right),
$$

where $\hat{\beta}$ is expressed by $\beta$ (defined in (3.11)),

$$
\hat{\beta}(\chi, z)=k_{1} \beta\left(k_{2}^{\prime} \chi, z\right)+k_{2} \beta\left(-k_{1}^{\prime} \chi, z\right) .
$$

The exterior derivative of the boundary two-form gives the induced $\mathrm{H}$-field, $\hat{H}=d \hat{\omega}$,

$$
\begin{aligned}
& \left(\hat{H}_{\chi^{i}}\right)_{a b}=\frac{k k_{1}^{\prime} k_{2}^{\prime}}{2}\left(\begin{array}{c|c|c}
0 & 0 \\
\hline & \frac{0}{-\beta,,_{i}\left(k_{2}^{\prime} \chi\right)+\beta,,_{i}\left(-k_{1}^{\prime} \chi\right)} & \beta,{ }_{i}\left(k_{2}^{\prime} \chi\right)-\beta,,_{i}\left(-k_{1}^{\prime} \chi\right) \\
0
\end{array}\right)+\mathcal{O}(z) \\
& \left(\hat{H}_{z^{m}}\right)_{a b}=\left(\begin{array}{c|c|c}
0 & * \\
\hline * & * & \square \\
\hline
\end{array}\right)+\mathcal{O}(z) \quad\left(\text { similarly for } \hat{H}_{\bar{z}^{m}}\right) .
\end{aligned}
$$

Now we have all geometric data at our disposal to compute the (generalised) second fundamental form that appears in the DBI equations of motion.

\subsection{Generalised second fundamental form}

The generalisation of the second fundamental form $\Omega_{a b}^{\mu}$ in the presence of a background $\mathrm{H}$-field is given in (1.8). As our embedding (3.1) is linear, the second derivatives $\partial_{a} \partial_{b} X^{\mu}$ vanish, and $\Omega_{a b}^{\mu}$ is just

$$
\Omega_{a b}^{\mu}=\Gamma_{\nu \lambda}^{\prime \mu} \partial_{a} X^{\nu} \partial_{b} X^{\lambda}-\hat{\Gamma}_{a b}^{c} \partial_{c} X^{\mu}=: \Omega_{a b}^{(T S) \mu}-\Omega_{a b}^{(W S) \mu} .
$$

In the following, we shall need the combinations

$$
\mathscr{G}_{i}^{ \pm}(y)=\left(\gamma, y^{i}(y, z) \pm \beta,_{y^{i}}(y, z)\right)_{z=0} .
$$

If $\mu$ corresponds to a torus direction, the quantities $\Omega^{(T S) \mu}$ and $\Omega^{(W S) \mu}$ have relatively simple expressions,

$$
\begin{aligned}
& \left(\Omega^{(T S) \mu}\right)_{a b}=\left(\Gamma_{\lambda \nu}^{\mu}-\frac{1}{2} H_{\lambda \nu}^{\mu}\right) \partial_{a} X^{\lambda} \partial_{b} X^{\nu}=G^{\rho \mu}\left(\Gamma_{\rho \lambda \nu}-\frac{1}{2} H_{\rho \lambda \nu}\right) \partial_{a} X^{\lambda} \partial_{b} X^{\nu} \\
& =\left\{\begin{array}{l}
-\frac{1}{2}\left(\begin{array}{l|c|c}
0 & \multicolumn{2}{|c}{0} \\
\hline 0 & 0 & \mathscr{G}_{i}^{+}\left(k_{2}^{\prime} \chi\right) \\
\left.\hline \mathscr{G}_{i}^{-}\right)^{T}\left(k_{2}^{\prime} \chi\right) & 0
\end{array}\right)+\mathcal{O}(z) \quad \text { for } \mu \cong \chi_{(1)}^{i} \\
-\frac{1}{2}\left(\begin{array}{c|c|c}
0 & \multicolumn{2}{|c|}{0} \\
\hline 0 & 0 & \mathscr{G}_{i}^{+}\left(-k_{1}^{\prime} \chi\right) \\
\left(\mathscr{G}_{i}^{-}\right)^{T}\left(-k_{1}^{\prime} \chi\right) & 0
\end{array}\right)+\mathcal{O}(z) \text { for } \mu \cong \chi_{(2)}^{i}
\end{array}\right.
\end{aligned}
$$


and

$$
\begin{aligned}
& \left(\Omega^{(W S) \mu}\right)_{a b}=\left(\hat{\Gamma}_{a b}^{c}-\frac{1}{2} \hat{H}_{a b}^{c}\right) \partial_{c} X^{\mu}=\left(\hat{\Gamma}_{n a b}-\frac{1}{2} \hat{H}_{n a b}\right) \hat{g}^{n c} \partial_{c} X^{\mu}
\end{aligned}
$$

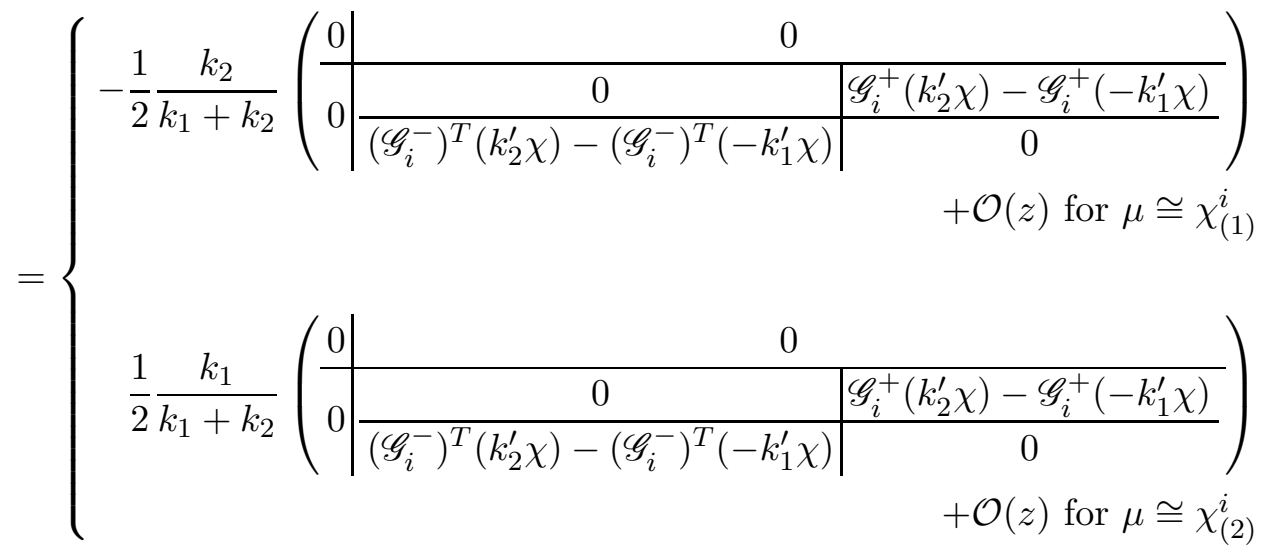

The expressions for $\Omega^{\mu}$ for non-toroidal directions $\mu$ are more involved, we however only need the block structure,

$$
\Omega^{\mu}=\left(\begin{array}{c|c|c}
0 & * \\
\hline * & * & \square \\
\hline & * & *
\end{array}\right)+\mathcal{O}(z) \quad \text { for } \mu \cong z^{m}, \bar{z}^{m}
$$

\subsection{Checking the equations of motion}

Now we have all the elements to check that the proposed geometry (1.1) satisfies the DBI equations of motion (1.6) and (1.7). An important quantity in the DBI equations is the sum $\hat{g}+\hat{\omega}$. From the equations (3.19) and (3.25) we find

$$
(\hat{g}+\hat{\omega})_{a b}=\left(\begin{array}{c|c}
{\left[\frac{k_{1}^{\prime} k_{2}^{\prime}}{2}\left(k_{1}+k_{2}\right)\right] \mathbb{1}_{r}} & \multicolumn{2}{|c}{0} \\
\hline 0 & \frac{1}{2}\left(\begin{array}{c|c}
\hline \hat{\mathcal{O}\left(z^{2}\right)} & \hat{\gamma}+\hat{\beta} \\
\hline \hat{\gamma}+\hat{\beta} & \mathcal{O}\left(z^{2}\right)
\end{array}\right)
\end{array}\right)
$$

The explicit expression for the constituent matrices is

$$
\frac{1}{2}(\hat{\gamma}+\hat{\beta})_{m n}=\left\{\begin{array}{lr}
k_{1}+k_{2}-\left(k_{1} e^{i k_{2}^{\prime}\left(\chi^{k} \alpha_{k}^{n}\right)}+k_{2} e^{-i k_{1}^{\prime}\left(\chi^{k} \alpha_{k}^{n}\right)}\right)+\mathcal{O}\left(z^{2}\right) & \text { for } m=n \\
\mathcal{O}(z) & \text { for } m \neq n
\end{array}\right.
$$

Notice again that there are no linear terms in $z$ on the diagonal, and that the off-diagonal terms are all at least of linear order with the property

$$
\left.\partial_{a}(\hat{\gamma}+\hat{\beta})_{m n}\right|_{z=0}=0 \quad \text { for } a \cong z^{m}, \bar{z}^{m}
$$


which follows from (3.7) and (3.12). The inverse of $(\hat{g}+\hat{\omega})$ that enters the DBI equations of motion is given by

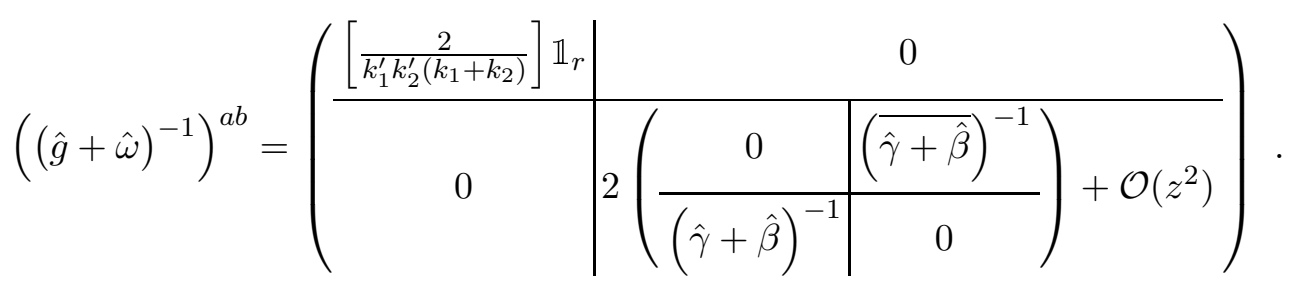

We are now prepared to check the equations of motion. Let us start with the equation coming from the variation of the gauge field.

\subsubsection{Gauge field equation of motion}

Let us recall the gauge field equation of motion (see (1.7)),

$$
\partial_{a}\left(\sqrt{\operatorname{det}(\hat{g}+\hat{\omega})}\left[(\hat{g}+\hat{\omega})^{-1}\right]_{\text {antisym }}^{a b}\right)=0 .
$$

From the explicit form (3.37) of $(\hat{g}+\hat{\omega})^{-1}$, we see that for toroidal directions, $b \cong \chi^{i}$, we have

$$
\left[(\hat{g}+\hat{\omega})^{-1}\right]_{\text {antisym }}^{a \chi^{i}}=0,
$$

so that the equation of motion (3.38) is satisfied for toroidal directions $b$.

For non-toroidal directions $b$, only derivatives in non-toroidal directions $a$ occur in the equation of motion (3.38). As it suffices to check the equation at $z=0$, we have to investigate the linear terms in $z$ in the expression in parentheses in (3.38). For the determinant of $\hat{g}+\hat{\omega}$ we have

$$
\operatorname{det}(\hat{g}+\hat{\omega}) \propto|\operatorname{det}(\hat{\gamma}+\hat{\beta})|^{2}+\mathcal{O}\left(z^{2}\right) .
$$

Now the matrix structure of $\hat{\gamma}+\hat{\beta}$ comes into play, which is of the form (see (3.35)),

$$
\hat{\gamma}+\hat{\beta}=D+N(z)+\mathcal{O}\left(z^{2}\right)
$$

where $D$ is a diagonal matrix independent of $z$ and $N(z)$ is off-diagonal and linear in $z$. This means that the determinant of $\hat{\gamma}+\hat{\beta}$ has no linear term - in the Leibniz sum formula for the determinant there is no summand that contains precisely one off-diagonal matrix element - and hence its derivative with respect to any $z^{m}$ vanishes at $z=0$.

It remains to discuss the linear terms in $\left[(\hat{g}+\hat{\omega})^{-1}\right]$. Its building blocks (see $(3.37)$ ) are given by the inverse of (3.41),

$$
(\hat{\gamma}+\hat{\beta})^{-1}=D^{-1}-D^{-1} N(z) D^{-1}+\mathcal{O}\left(z^{2}\right) .
$$

The linear terms in $(\hat{\gamma}+\hat{\beta})^{-1}$ are all off-diagonal, and to leading order the off-diagonal elements are proportional to the off-diagonal elements of $\hat{\gamma}+\hat{\beta}$, so that the property (3.36) carries over to $(\hat{\gamma}+\hat{\beta})^{-1}$,

$$
\left.\partial_{a}\left((\hat{\gamma}+\hat{\beta})^{-1}\right)^{m n}\right|_{z=0}=0 \quad \text { for } a \cong z^{m}, \bar{z}^{m}
$$


From this we can finally conclude ${ }^{3}$ that the equation of motion (3.38) is also satisfied for non-toroidal directions $b$.

\subsubsection{Embedding equations of motion}

It remains to check the equation of motion (1.6) that comes from the variation of the embedding of the brane,

$$
\left[(\hat{g}+\hat{\omega})^{-1}\right]^{b a} \Omega_{a b}^{\mu}=0 .
$$

For non-toroidal directions $\mu$, the block structure of the matrices that are involved is enough to verify the equation of motion,

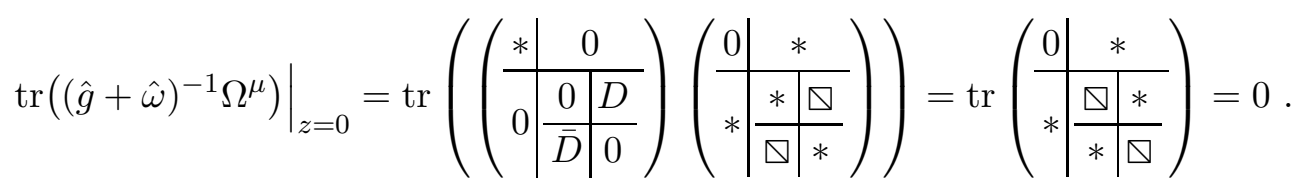

Here, $D$ is a placeholder for an arbitrary diagonal matrix, and $\nabla$ for any off-diagonal matrix.

For a toroidal direction, $\mu \cong \chi_{(i)}^{l}(i=1,2)$, one needs the explicit expressions for the matrices,

$$
\left.(\Omega)_{a b}^{\mu}\right|_{z=0}=-\frac{1}{2}\left(\begin{array}{c|c|c}
0 & \multicolumn{2}{|c}{0} \\
\hline 0 & 0 & \Delta^{\mu} \\
\hline \bar{\Delta}^{\mu} & 0
\end{array}\right)
$$

with

$$
\begin{aligned}
\Delta_{m n}^{\mu} & =\frac{k_{1}}{k_{1}+k_{2}}\left(\mathscr{G}_{l}^{+}\right)_{m n}\left(k_{2}^{\prime} \chi\right)+\frac{k_{2}}{k_{1}+k_{2}}\left(\mathscr{G}_{l}^{+}\right)_{m n}\left(-k_{1}^{\prime} \chi\right) \\
& =\frac{2 i \alpha_{l}^{n}}{k_{1}+k_{2}}\left[k_{1}+k_{2}-\left(k_{1} e^{i k_{2}^{\prime}\left(\chi^{k} \alpha_{k}^{n}\right)}+k_{2} e^{-i k_{1}^{\prime}\left(\chi^{k} \alpha_{k}^{n}\right)}\right)\right] \delta_{m n} \quad \text { for } \mu \cong \chi_{(1,2)}^{l} .
\end{aligned}
$$

Comparing this result with the expressions (3.35) that we got for the constituent matrices $\hat{\gamma}+\hat{\beta}$ of $\hat{g}+\hat{\omega}$, we find the relationship

$$
\Delta_{m n}^{\mu}=\frac{i \alpha_{l}^{n}}{k_{1}+k_{2}}(\hat{\gamma}+\hat{\beta})_{m n}+\mathcal{O}(z) \text { for } \mu \cong \chi_{(1,2)}^{l} .
$$

This is the crucial property that helps us to verify the equations of motion (3.44):

$$
\begin{aligned}
& \left.\left.\operatorname{tr}\left[(\hat{g}+\hat{\omega})^{-1} \Omega^{\mu}\right]\right|_{z=0}=-\operatorname{tr}\left[\begin{array}{c|c|c|c|c|c|c|c|}
{\left[\frac{1}{k_{1}^{\prime} k_{2}^{\prime}\left(k_{1}+k_{2}\right)}\right] \mathbb{1}_{r}} & 0 \\
\hline 0 & \left(\begin{array}{c|c|c}
0 & \hat{\gamma}+\hat{\beta} \\
\hline \hat{\gamma}+\hat{\beta} & 0
\end{array}\right)^{-1}
\end{array}\right)\left(\begin{array}{c|c|c}
0 & 0 \\
\hline 0 & 0 & \Delta^{\mu} \\
\hline \bar{\Delta}^{\mu} & 0
\end{array}\right)\right]_{z=0} \\
& =-\frac{1}{k_{1}+k_{2}} \operatorname{tr}\left[\left(\begin{array}{c|c}
\left(i \alpha_{l}^{n} \delta_{m n}\right)_{m n} & 0 \\
\hline 0 & \left(-i \alpha_{l}^{n} \delta_{m n}\right)_{m n}
\end{array}\right)\right] \quad\left(\mu \cong \chi_{(1,2)}^{l}\right) \\
& =0 \text {. }
\end{aligned}
$$

This finally shows that the generalised permutation brane given by the embedding (1.1) and the boundary two-form $\hat{\omega}$ stated in (1.5) is a solution of the DBI equations of motion.

\footnotetext{
${ }^{3}$ The gauge field equations of motion were also investigated in [8], and for the toroidal directions the correct argument was already given there. For the non-toroidal directions, however, it was implicitly assumed that already the matrix $\hat{\gamma}+\hat{\omega}$ has no linear terms in $z$ which is in general not correct.
} 


\section{$4 \quad$ Further directions}

We have shown in this paper that the generalised permutation branes are solutions of the DBI equations for products $G_{k_{1}} \times G_{k_{2}}$ with an arbitrary compact simply connected, simple Lie group $G$. A natural extension of this work would be to investigate generalised permutation D-branes in products of coset models. As mentioned in the introduction, such generalisations have been formulated in the Landau-Ginzburg description of $N=2$ minimal models, which can also be described as cosets $S U(2) / U(1)$, their geometric interpretation is however unclear. Proposals for the geometries of such D-branes in coset models $G / H$ have been made in $[8,18]$, but it is questionable whether any of these proposals is correct, as no successful DBI analysis could be carried out so far. Another approach to find the geometries of these branes in products like $S U(2) / U(1) \times S U(2) / U(1)$ is by starting with generalised permutation branes on $S U(2) \times S U(2)$, and then marginally perturb the $S U(2)$ 's by currentcurrent deformations (see e.g.[19]). At the end-point of the deformation one expects a decoupling of one dimension and the $S U(2)$ 's reduce essentially to cosets $S U(2) / U(1)$ (see e.g.[20]). In general it can happen that the branes start to flow when the background is deformed (see e.g.[21]), but it is conceivable that it is possible to tune the deformations of the two factors such that the generalised permutation brane does not flow. This is currently under investigation.

\section{Acknowledgments}

We thank Sebastian Krug and Rafał Suszek for interesting and useful discussions.

Open Access. This article is distributed under the terms of the Creative Commons Attribution Noncommercial License which permits any noncommercial use, distribution, and reproduction in any medium, provided the original author(s) and source are credited.

\section{References}

[1] A. Recknagel, Permutation branes, JHEP 04 (2003) 041 [hep-th/0208119] [SPIRES].

[2] J.M. Figueroa-O'Farrill and S. Stanciu, D-branes in $A d S_{3} \times S^{3} \times S^{3} \times S^{1}$, JHEP 04 (2000) 005 [hep-th/0001199] [SPIRES].

[3] M.R. Gaberdiel and S. Schäfer-Nameki, D-branes in an asymmetric orbifold, Nucl. Phys. B 654 (2003) 177 [hep-th/0210137] [SPIRES].

[4] G. Sarkissian and M. Zamaklar, Symmetry breaking, permutation D-branes on group manifolds: boundary states and geometric description, Nucl. Phys. B 696 (2004) 66 [hep-th/0312215] [SPIRES].

[5] T. Quella, On the hierarchy of symmetry breaking D-branes in group manifolds, JHEP 12 (2002) 009 [hep-th/0209157] [SPIRES].

[6] S.K. Ashok, E. Dell'Aquila and D.-E. Diaconescu, Fractional branes in Landau-Ginzburg orbifolds, Adv. Theor. Math. Phys. 8 (2004) 461 [hep-th/0401135] [SPIRES].

[7] I. Brunner and M.R. Gaberdiel, Matrix factorisations and permutation branes, JHEP 07 (2005) 012 [hep-th/0503207] [SPIRES]. 
[8] S. Fredenhagen and T. Quella, Generalised permutation branes, JHEP 11 (2005) 004 [hep-th/0509153] [SPIRES].

[9] V. Braun and S. Schäfer-Nameki, D-brane charges in Gepner models, J. Math. Phys. 47 (2006) 092304 [hep-th/0511100] [SPIRES].

[10] C. Caviezel, S. Fredenhagen and M.R. Gaberdiel, The RR charges of A-type Gepner models, JHEP 01 (2006) 111 [hep-th/0511078] [SPIRES].

[11] S. Fredenhagen and M.R. Gaberdiel, Generalised $N=2$ permutation branes, JHEP 11 (2006) 041 [hep-th/0607095] [SPIRES].

[12] E.S. Fradkin and A.A. Tseytlin, Nonlinear electrodynamics from quantized strings, Phys. Lett. B 163 (1985) 123 [SPIRES].

[13] A. Abouelsaood, C.G. Callan, Jr., C.R. Nappi and S.A. Yost, Open strings in background gauge fields, Nucl. Phys. B 280 (1987) 599 [SPIRES].

[14] C.G. Callan, Jr., C. Lovelace, C.R. Nappi and S.A. Yost, String loop corrections to $\beta$-functions, Nucl. Phys. B 288 (1987) 525 [SPIRES].

[15] S. Ribault, D3-branes in NS5-branes backgrounds, JHEP 02 (2003) 044 [hep-th/0301092] [SPIRES].

[16] J.M. Maldacena, G.W. Moore and N. Seiberg, D-brane instantons and K-theory charges, JHEP 11 (2001) 062 [hep-th/0108100] [SPIRES].

[17] T. Bröcker and T. Dieck, Representations of compact Lie groups, Springer U.S.A. (1985).

[18] G. Sarkissian, Generalised permutation branes on a product of cosets $G\left(k_{1}\right) / H \times G\left(k_{2}\right) / H$, Nucl. Phys. B 747 (2006) 423 [hep-th/0601061] [SPIRES].

[19] A. Giveon and E. Kiritsis, Axial vector duality as a gauge symmetry and topology change in string theory, Nucl. Phys. B 411 (1994) 487 [hep-th/9303016] [SPIRES].

[20] S. Förste, D-branes on a deformation of SU(2), JHEP 02 (2002) 022 [hep-th/0112193] [SPIRES].

[21] S. Fredenhagen, M.R. Gaberdiel and C.A. Keller, Bulk induced boundary perturbations, J. Phys. A 40 (2007) F17 [hep-th/0609034] [SPIRES]. 